\title{
Review Article \\ The Characteristics of Thrombin in Osteoarthritic Pathogenesis and Treatment
}

\author{
Pei-Yu Chou, ${ }^{1,2}$ Chen-Ming Su, ${ }^{1}$ Chun-Yin Huang, ${ }^{3,4}$ and Chih-Hsin Tang ${ }^{1,5,6}$ \\ ${ }^{1}$ Graduate Institute of Basic Medical Science, China Medical University, No. 91, Hsueh-Shih Road, Taichung, Taiwan \\ ${ }^{2}$ Department of Nursing, Hung Kuang University, Taichung, Taiwan \\ ${ }^{3}$ Department of Orthopaedic Surgery, China Medical University Beigang Hospital, Yun-Lin County, Taiwan \\ ${ }^{4}$ Graduate Institute of Clinical Medical Science, China Medical University, Taichung, Taiwan \\ ${ }^{5}$ Department of Pharmacology, School of Medicine, China Medical University, Taichung, Taiwan \\ ${ }^{6}$ Department of Biotechnology, College of Health Science, Asia University, Taichung, Taiwan
}

Correspondence should be addressed to Chih-Hsin Tang; chtang@mail.cmu.edu.tw

Received 9 July 2014; Accepted 1 September 2014; Published 16 September 2014

Academic Editor: Wen-Bin Wu

Copyright (C) 2014 Pei-Yu Chou et al. This is an open access article distributed under the Creative Commons Attribution License, which permits unrestricted use, distribution, and reproduction in any medium, provided the original work is properly cited.

Osteoarthritis $(\mathrm{OA})$ is a mechanical abnormality associated with degradation of joints. It is characterized by chronic, progressive degeneration of articular cartilage, abnormalities of bone, and synovial change. The most common symptom of OA is local inflammation resulting from exogenous stress or endogenous abnormal cytokines. Additionally, OA is associated with local and/or systemic activation of coagulation and anticoagulation pathways. Thrombin plays an important role in the stimulation of fibrin deposition and the proinflammatory processes in OA. Thrombin mediates hemostatic and inflammatory responses and guides the immune response to tissue damage. Thrombin activates intracellular signaling pathways by interacting with transmembrane domain G protein coupled receptors (GPCRs), known as protease-activated receptors (PARs). In pathogenic mechanisms, PARs have been implicated in the development of acute and chronic inflammatory responses in OA. Therefore, discovery of thrombin signaling pathways would help us to understand the mechanism of OA pathogenesis and lead us to develop therapeutic drugs in the future.

\section{Introduction}

Osteoarthritis (OA) is a mechanical abnormality associated with degradation of joints, including articular cartilage, synovial fluid, and subchondral bone $[1,2]$. As per the American College of Rheumatology, $70 \%$ of people over the age of 70 years have X-ray evidence of OA [3]. Patients with OA initially begin with adequate fluid content and healthy cartilage but gradually deteriorate over time, showing progressively decreased joint composition. Therefore, OA is termed as a degenerative joint disease [4]. Since several factors can contribute to development of $\mathrm{OA}$, such as overuse of joints or exogenous stress, anyone can develop the disease [5].

\section{Osteoarthritis}

$\mathrm{OA}$ is characterized as a chronic, progressive degeneration of articular cartilage, abnormalities of bone, and synovial changes. Typical characteristics of OA include progressive loss of articular cartilage resulting in structural and functional failure of joints. Since cartilage plays an important role as a cushion within the joint, loss of articular cartilage causes intolerable pain. The most common symptom of OA is local inflammation resulting from exogenous stress or endogenous, abnormal cytokines [6,7]. Normal cartilage matrix is mainly composed of type II collagen, which provides tensile support for the tissue [8], in addition to proteoglycans, and chondrocytes [9]. It has been reported that matrix metalloproteinase (MMP) is overexpressed in patients with OA resulting in cleavage of collagen and proteoglycans from the matrix [10]. In addition, numerous studies have verified abnormal synovium within the OA joint as compared to normal synovium, which is shown adequate blood and nerve supply. These abnormalities include thickening of the lining layer, increased vascularity, inflammatory cell infiltration 
leading to local synovitis, hypertrophy, and thickening of the joint capsule [11]. Treatment options for OA focus on pain relief and reducing inflammation. Therefore, traditional treatment for OA includes nonsteroidal anti-inflammatory drugs (NSAID), analgesics, and steroid injections that are used to treat pain and inflammation $[12,13]$. Since the exact etiology of OA is not well understood, biochemical markers could help us better understand the pathogenesis of OA and design new therapeutic approaches for the treatment of the OA.

\section{Characteristics of Thrombin}

Thrombin, also known as blood-coagulation factor IIa (FIIa), and its inactive precursor prothrombin, also called coagulation factor II (FII), are serine proteases and members of the family of vitamin K-dependent coagulation factors. The zymogen prothrombin is enzymatically cleaved by the prothrombinase complex through the activated platelet phospholipids factor Xa (FXa) and factor Va (FVa) [14, 15]. Thrombin is essential for homeostasis, thrombosis, and inflammation triggered by tissue damage. Thrombin has two important functions: coagulation and anticoagulation. Additionally, arthritis is involved in local and/or systemic activation of coagulation and anticoagulation pathways. However, during coagulation, thrombin present in the blood can result in widespread thrombosis and cause a reduction in blood flow [16]. Bokarewa and colleagues demonstrated that tissue-factor (TF) is expressed in endothelial cells producing tumor necrosis factor (TNF) and interleukin-1 (IL-1) and in monocytes inducing chemokines, such as macrophage inflammatory protein 1 (MIP-1) and chemokine (C-C motif) ligand 5 (CCL5) $[17,18]$. Thus, TF is considered to appear as a result of inflammation and triggers the immune response and coagulation systems. Thrombin is not only a mitogen but also a potent vasoconstrictor, causing local vasoconstriction, which directly stimulates vascular smooth muscle and adrenergic receptors. Thrombin is involved in tissue repair, activation of platelet and endothelial cells, and inflammation by stimulating deposition of fibrin. As part of its coagulation function, thrombin stimulates fibrin deposition, thus influencing inflammation, and activates transglutaminase factor XIIIa to convert soluble fibrinogen into an insoluble fibrin clot [19]. Fibrin is a ligand for intercellular adhesion molecule-1 (ICAM-1, CD54), CD11b/CD18 (CR3, Mac-1), and CD11c/CD18 (CR4, p150/95). Fibrin binds to $\alpha / \beta$ integrins to promote adhesion and migration of leucocytes, followed by accumulation of leukocytes in the matrix during inflammation [20-22]. Direct injection of thrombin has been shown to stimulate peritoneal accumulation of IL- 6 and MCP-1 in a fibrin-dependent manner [23]. Thus, thrombin regulates fibrin to induce chemokines/cytokines during inflammation.

Thrombin participates in anticoagulation, also called fibrinolysis, during the process of inflammation by forming a complex with thrombomodulin (TM), an integral membrane protein expressed in endothelial cells. The thrombin-TM complex activates protein $\mathrm{C}$ (PC), an inhibitor of the coagulation cascade, which binds to protein $\mathrm{S}$, and, in turn, this stops the process of coagulation. Besides, this complex also activates thrombin activatable fibrinolysis inhibitor (TAFI) to prolong clot lysis and inhibit coagulation via removing terminal lysine residues from fibrin [24, 25]. For TF-induced coagulation, thrombin also activates TF pathway inhibitor (TFPI) by inhibiting prothrombinase complexes from initiating coagulation [26-29]. Hence, thrombin-TM complex also inhibits fibrinolysis by the profibrinolytic effect of anticoagulation proteases activated protein C (APC), which causes further anticoagulation. Overall, thrombin does not simply act as a mediator of coagulation or anticoagulation, but rather as a signaling molecule to control inflammation.

\section{Thrombin-Dependent Signaling and Protease-Activated Receptors}

Thrombin promotes platelet activation and aggregation via activation of protease-activated receptors (PARs) on the platelet surface. Thrombin activates intracellular signaling pathways by interacting with transmembrane domain G protein coupled receptors (GPCRs), also known as PARs. There are four members of the PAR family, namely, PAR-1, PAR-2, PAR-3, and PAR-4. Thrombin activates PAR-1, PAR-3, and PAR-4, but not PAR-2 $[16,30]$. Thrombin or serine proteinases activate also PARs by cleaving an $\mathrm{N}$-terminal peptide bond and generating a new $\mathrm{N}$-terminus, which acts as a ligand for transmembrane receptors to induce signal transduction [31-33]. Thus, PARs undergo conformational changes to couple with heterotrimeric $\mathrm{G}$ proteins, of which, PAR-1 couples with $G$ protein subtypes, such as $G \alpha_{q}, G \alpha_{12 / 13}$, and $G \alpha_{i}$ to activate mitogen-activated protein kinase (MAPK) cascades (Table 1) $[31,34,35]$. PAR-1 is also activated by coagulation FXa as well as APC, matrix metalloproteinase-1 (MMP-1), neutrophil elastase (NE), and neutrophil proteinase-3 (PR3) [31]. PAR-3 and PAR-4 are mainly activated by thrombin, but PAR- 4 can also be activated by cathepsin G, a protease secreted by neutrophils $[35,36]$. Although PAR-3 acts as a cofactor binding to thrombin to activate PAR- 4 in rat, its mechanism of action in humans remains unclear [31, 35, 37]. PAR-2 is insensitive to thrombin; however, it can be activated by serine proteinases, mast cell tryptase, and allergic or bacterial proteases. Arrestin, a PAR2-selective agonist, is suggested to support extracellular regulated kinases $1 / 2$ (ERK1/2) signaling in the cytoplasm, independently of Gprotein activation $[35,38]$.

PARs are not only highly expressed on platelets, but also found on endothelial cells, monocytes, fibroblasts, Tlymphocytes, smooth muscle cells, and certain tumor cells $[16,31]$. Activation of PARs on platelets is critical for hemostasis and thrombosis. For endothelial cells, thrombin-induced endothelial cell hyperpermeability results in recruitment of immune cells and release of growth factors and cytokines $[39,40]$. Thrombin also induces release of calcium through RhoA/Rho kinase pathway and activation of myosin light chain (MLC) kinase to inhibit myosin phosphatase, causing a disruption of endothelial barrier function through interactions with actin-myosin $[16,34]$. Thrombin-induced protein-tyrosine kinase (PTK) affects the phosphorylation 
TABLE 1: Thrombin as an inflammatory-related molecule regulates signaling in OA.

\begin{tabular}{|c|c|c|c|c|}
\hline Receptor & Regulation & Pathway & Function & Reference \\
\hline PAR-1 & CCL2 & PKC/c-SRC/EGFR/MEK/ERK/AP-1 & $\begin{array}{l}\text { Monocytes, memory } \mathrm{T} \text { cells, and } \\
\text { dendritic cells activation }\end{array}$ & {$[47,48]$} \\
\hline PAR-1/-2 & IL-1, TNF- $\alpha$ & $\mathrm{p} 38 / \mathrm{p} 42 / 44$ & MMP expression & {$[65]$} \\
\hline PAR-1/-3 & $\mathrm{HO}-1$ & $\mathrm{PKC} \delta / \mathrm{c}-\mathrm{SRC} / \mathrm{Nrf}-2$ & Synovial fibroblasts inflammatory & {$[70-72]$} \\
\hline PAR-1/-3 & MMP-13 & 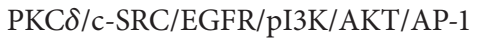 & Cartilage destruction & {$[59,60]$} \\
\hline PAR-1 & COX-2 & $?$ & $\begin{array}{l}\text { IL-6, CTGF, FGF1/2, VEGF, Tnc } \\
\text { expression }\end{array}$ & {$[51-54]$} \\
\hline
\end{tabular}

PAR: protease-activated receptor; CCL2: chemokine (C-C motif) ligand-2; IL: interleukin; TNF- $\alpha$ : tumor necrosis factor-alpha; VEGF: vascular endothelial growth factor; MMP: matrix metalloproteinase; FGF-1/-2: fibroblast growth factor-1/-2; CTGF: connective tissue growth factor; TnC: tenascin C; COX-2: cyclooxygenase- 2 .

of junctional proteins, vascular endothelial-cadherin (VEcadherin), and catenins and results in migration and proliferation in several tumors such as lung, colon, and gastric cancer [35, 41-43]. Previous studies have implicated PARs in the development of acute and chronic inflammatory responses as well as thrombin [16, 31, 35]. The role of PAR-1 in inflammation has been clearly established, in vasodilation and mast cell degranulation, which increase the production of cytokines or chemokines via PAR-1, and in the adhesion of macrophage via fibrin [23]. Thrombin-regulated chemokine production also triggers calcium release in monocytes [44]. Stimulation of isolated monocytes by thrombin or a receptorselective PAR1-AP (TFLLRNPNDK) causes upregulation of monocyte chemotactic protein-1 (MCP-1) [44] or release of IL-6, respectively $[45,46]$. Taken together, these results suggest that thrombin mediates hemostatic and inflammatory responses and guides the immune response to tissue damage. Therefore, thrombin receptors involved in regulation of signaling cascades may provide a therapeutic target for inflammation.

\section{Thrombin as a Signaling Factor Regulating OA Progression}

Although OA is not considered systemic inflammatory disease, joint inflammation resulting from a local increase in synovial membrane vascularity or cartilage degradation is commonly associated with OA. OA is usually classified as a noninflammatory disorder. However, Thrombin is known to be involved in the regulation of fibrin deposition, cell migration, cell invasion, and proinflammatory processes. A number of growth factors and chemokines/cytokines have been found to regulate the expression of thrombin in many pathological states, indicating a critical role in OA progression. Therefore, we discuss different key events during OA pathogenesis, including cartilage degradation, synovial membrane abnormalities, chemokine/cytokine cascades, and production of inflammatory mediators such as heme oxygenase (HO).

5.1. Chemokines/Cytokines. Numerous chemokines/cytokines have been found to regulate expression of thrombin in OA [23]. Chemokine (C-C motif) ligand-2 (CCL2)/MCP-1 are known to be expressed in $\mathrm{OA}$ and rheumatoid arthritis
[47, 48]. CCL2 recruits monocytes, memory T cells, and dendritic cells to the site of inflammation that is triggered by tissue damage [49]. Recently, expression of CCL2 has been detected in pathological arthritis via macrophage aggregation [50]. A study further suggested that thrombin induced CCL2 production via the PAR-1 mediated c-Src/MEK/ERKdependent signaling pathway in human osteoblasts (Table 1) [48]. Thrombin also increased the expression of inflammatory cytokines such as IL- $1 \beta$, TNF- $\alpha$, fibroblast growth factor-1/-2 (FGF-1/-2), transforming growth factor- $\beta 1$ (TGF$\beta 1$ ), IL-6, connective tissue growth factor (CTGF), tenascin $\mathrm{C}(\mathrm{TnC})$, and cyclooxygenase-2 (COX-2) in osteoblasts or osteoblast-like cells (Table 1) [51-54]. Thus, thrombin was shown to induce the release of proinflammatory cytokines via PAR-1 on the surface of osteoblasts. However, Pagel and colleagues found that earlier stages of bone repair are delayed in mice lacking PAR-1 [55]. Taken together, these results suggest that thrombin-induced activation of various chemokines/cytokines via PAR-1 may be one key mechanism of inflammation in OA.

5.2. Cartilage. It is necessary to conserve structural and functional integrity of the bone tissue during normal physiological conditions. Chondrocytes can maintain equilibrium between anabolic and catabolic activities in cartilage [10]. It is not yet completely understood which factors are responsible for initiating the degradation and loss of articular tissue. Thrombin is a key mediator to release proteoglycan in the degradation of human and bovine cartilage [56]. In $\mathrm{OA}$, chondrocytes increase production of various proteolytic enzymes such as aggrecanases and MMP, resulting in aberrant cartilage destruction [10]. It has been shown previously that MMP-13 is overexpressed in OA $[57,58]$, which caused degradation of articular cartilage components, such as types II, IV, and IX collagen and proteoglycan [59]. Shiomi et al. showed that specific deletion of MMP-13 (gene) or inhibition of MMP-13 activity in cartilage decelerated aggressive OA (Table 1) [59]. Therefore, Huang et al. investigated the intracellular signaling pathways involved in thrombininduced MMP-13 expression using human chondrocytes, which showed reduced MMP-13 production after transfection with siRNAs against PAR1 and PAR3, but not PAR4. The result also found that PAR1/PAR3 receptors activation by 
MMP-13 is increased by thrombin via a mechanism involving EGFR transactivation and activation of $\mathrm{PKC} \delta, \mathrm{c}-\mathrm{Src}, \mathrm{PI} 3 \mathrm{~K}$, Akt, and finally AP-1 on the MMP-13 promoter, thereby contributing to cartilage destruction during arthritis [60]. $\mathrm{HO}$ is another inflammatory mediator consisting of three isoforms: $\mathrm{HO}-1, \mathrm{HO}-2$, and a less-characterized HO-3 [61]. $\mathrm{HO}-1$ is inducible in response to stress such as reactive oxygen species, nitric oxide, heat shock, and hypoxia, and it also protects against oxidative injury and decreases inflammation $[62,63]$. It has reported that $\mathrm{HO}-1$ could inhibit cartilage erosion accompanied by extensive fibrosis in the joint [64]. In animal arthritis models, the levels of TNF- $\alpha$, IL- 2 , and IL-10 were decreased by the HO-1 inducer cobalt protoporphyrin IX (CoPP), indicating that HO-1 deficiency causes chronic inflammatory conditions in arthritis. Moreover, HO1 induced by the clinical vasodilator, sodium nitroprusside, inhibits apoptosis of arthritic chondrocytes through ERK inhibition and p38 activation to decrease activity of MMP-1 and MMP-13 (Table 1) [65]. These findings show that thrombin-activated cartilage destruction in OA pathogenesis occurs via MMP-13.

5.3. Synovium. Once OA onset occurs, macrophages can invade or migrate into a joint. The macrophages then release large amounts of proinflammatory and procatabolic mediators into the synovium [66]. In addition, thrombinderived from the synovial fluids of OA patients also mediated production of proinflammatory factors and was characterized as a marker of synovitis [67]. Synovitis is secondary to cartilage degradation, which occurs in the adjacent, damaged cartilage. This inflammation is characterized by large amounts of proinflammatory and procatabolic mediators and a local increase in synovial membrane vascularity. In advanced OA, synovitis extends into the synovial membrane and progresses to fibrosis and villi hypertrophy [66]. In synovial cells, HO-1 is an important regulator of inflammation and affects cartilage degradation. Synovial membrane from OA patients has been found to produce IL$1 \beta$ and TNF- $\alpha$. Presence of chronic inflammatory factors and proinflammatory cytokines are a feature of synovial membranes from patients with early OA $[68,69]$. However, the exact mechanism of macrophage-derived proinflammatory cytokine production in arthritic synovium is not well understood. Previous studies found that OA synovial fibroblasts (OASFs) showed significantly higher expression of thrombin than normal synovial fibroblasts. Furthermore, it was shown that thrombin induced concentration- and time-dependent expression of $\mathrm{HO}-1$ in OASFs via PAR1 , PAR-3, and PKC $\delta / c-S r c$ signaling pathways [70, 71]. In pharmacologic inhibitors, thrombin-regulated HO-1 expression was attenuated by thrombin inhibitor, D-phenylalanylL-prolyl-L-arginine chloromethyl ketone (PPACK), PKC $\delta$ inhibitor (rottlerin), or c-Src inhibitor (PP2), suggesting a hint that thrombin is involved in upregulation of $\mathrm{HO}-$ 1. Besides, expression of nuclear factor erythroid-2-related factor (Nrf2) also contributes to thrombin-induced HO1 production in OASFs (Table 1) [72]. Consequently, the discovery of thrombin-mediated HO-1 expression clarified the mechanism of OA pathogenesis and may lead to the development of more effective therapeutic targets for OA treatment in the future.

\section{Thrombin as a Therapeutic Target in OA}

Given the important role of thrombin-mediated signaling in $\mathrm{OA}$ progression, there has been increasing interest in therapeutic strategies to target this protein. In the cartilage or synovium, increased expression of thrombin and downstream intracellular signaling pathway via transmembrane domain GPCRs, PARs, are closely associated with inflammation and its mediators. Signaling via PARs may also promote OA progression by inducing inflammation in osteoblasts, chondrocytes, and OASFs. Moreover, some studies indicate that thrombin acts as a mitogen to stimulate the abnormal proliferation of synovial cells during OA pathogenesis [73, 74]. The aim of this review is to summarize the mechanism of thrombin-mediated OA pathogenesis and develop therapeutic approaches for treatment of OA. These include thrombin antagonists and herbal medicines.

6.1. Thrombin Antagonists. Thrombin promotes fibrin formation and protein $\mathrm{C}$ activation. Factor XIII is activated by thrombin that stabilizes fibrin complex and stimulates platelet, which helps in clotting. On the other hand, thrombin interacts with thrombomodulin and activates protein $\mathrm{C}$ and TAFI to initiate anticoagulation and inhibit fibrinogenesis. Given its broad-spectrum activities, thrombin represents a good target for anticoagulant drugs such as heparin, warfarin, and direct thrombin inhibitors (DTIs) $[75,76]$. Thrombin has three binding sites: the active site, exosite 1 , and exosite 2 . The exosite 1 is anion-binding and binds to fibrin and exosite 2 is a heparin-binding domain $[75,77]$. Some drugs bind to either exosite 1 or exosite 2 and may influence activity at the active site. DTIs are anticoagulant drugs that are commonly used to prevent the blood clot formation by directly inhibiting thrombin. DTIs inhibit thrombin via two mechanisms: (1) where bivalent DTIs simultaneously block the active site and exosite 1, thus acting as competitive inhibitors of fibrin, and (2) where univalent DTIs block only the active site; both types of DTIs inhibit unbound and fibrin-bound thrombin [78]. Pradaxa (Dabigatran) and Acova (Argatroban), both univalent DTIs, are used mostly in cases of cardiovascular disease or its complications [76]. However, bleeding is the most common and serious side effect of DTIs. Thus far, DTIs are being developed, although researchers have recently focused on PAR-1 inhibition. Certain studies have reported thrombin inhibition via PAR-1-mediated platelet activation without increasing bleeding in preclinical models and smallscale clinical trials $[79,80]$. Patients with acute coronary syndromes that used PAR1 antagonist, Vorapaxar (SCH530348), showed reduced risk of cardiovascular death or ischemic events in two large phase III clinical trials (Table 2). Recently, another PAR-1 antagonist, Atopaxar (E5555) used in the guinea pig model and in clinical trials, showed inhibition of PAR-1-dependent platelet aggregation and coagulation (Table 2) [81, 82]. Nevertheless, thrombin is still being studied 
TABLE 2: Thrombin as a therapeutic target in OA.

\begin{tabular}{lllc}
\hline & Drug & Target & Reference \\
\hline \multirow{3}{*}{ Thrombin antagonists } & Vorapaxar (SCH530348) & PAR1 antagonist & {$[81,82]$} \\
& Atopaxar (E555) & PAR1 antagonist & {$[81,82]$} \\
& Hirudin & Cleave fibrinogen and PAR-1 & {$[74,83-85]$} \\
\hline \multirow{2}{*}{ Curcuminoids } & Anti-inflammatory & {$[88,89]$} \\
& & Chondrocytes protection & {$[88]$} \\
& & Bax and p53 reduction & {$[90]$} \\
Herbal medicines & Increase Bcl-xL expression, & {$[90]$} \\
& & Inhibited NF- $\kappa$ B phosphorylation, & {$[90]$} \\
& & I B $\alpha$ degradation, MMP-3, & {$[90]$} \\
& Ceiba pentandra & MMP-9, and COX-2 expression & {$[90]$} \\
& (Malvaceae/Bombacoideae) & Coagulation intrinsic pathway & {$[91]$} \\
& Quassia africana & Prolong clotting time & {$[91]$} \\
& (Simaroubaceae/C. pentandra) & Coagulation intrinsic pathway & {$[91]$} \\
\end{tabular}

for its relationship with local and systemic activation of coagulation and fibrinolysis pathways owing to thrombindependent fibrin generation and fibrin accumulation during OA pathogenesis [73]. In addition, expression of fibrin enhances inflammation and promotes cell adhesion and migration. Although the study pointed out that mediation of thrombin-induced inflammation via PAR-1 occurred in PAR-1 deficient mice, inhibition of PAR-1 had no effect on thrombin-dependent fibrin generation and coagulation [23]. However, Szaba and Smiley defined the roles for thrombin, PAR-1, and fibrinogen in a mouse peritonitis model [23]. They demonstrated that direct injection of thrombin can stimulate macrophage adhesion and peritoneal accumulation of cytokines in a fibrinogen-dependent manner in vivo. In further experiments with PAR-1-deficient mice, they found that thrombin stimulated vasoconstriction. Thrombin probably has pleiotropic functions, including PAR-1-mediated vasodilation, fibrin-activating macrophage adhesion, and cytokines/chemokines production during the inflammatory process [23]. In addition, Hirudin is the most potent natural inhibitor of thrombin, which possesses a specific activity to bind with the active site and fibrinogen-binding exosite 1 of thrombin and cleave fibrinogen and PAR-1 (Table 2) [83]. Some studies suggest that administration of Hirudin analogs can prevent onset and ameliorate arthritis by reducing leukocyte infiltration in a mouse glomerulonephritis model $[74,84$, 85]. Taken together, the potential applications of thrombin antagonists should be explored in the treatment of OA.

6.2. Herbal Medicines. Many studies have demonstrated that proinflammatory cytokines and anti-inflammatory cytokines such as, IL-1, IFN- $\gamma$, IL-6, IL-7, IL-10, and TNF- $\alpha$ are expressed in OA joints [86, 87]. Curcumin (diferuloylmethane) is an anti-inflammatory, polyphenolic phytochemical. The early degenerative changes in chondrocytes were relieved by curcumin when administered as cotreatment with IL-1 $\beta$. Additionally, collagen type II and $\beta 1$-integrin synthesis by IL- $1 \beta$ were inhibited by curcumin [88]. Clinical studies have also shown therapeutic efficacy of curcuminoids in OA patients, where significant improvements were noted in scores for pain and physical function [89]. Therefore, curcuminoids have well-known anti-inflammatory properties and exert protective effect on chondrocytes (Table 2). $\beta$ Ecdysterone (Ecd), a major component of Chinese herbal medicines, is an estrogen analog, which was shown to protect chondrocytes from IL- $1 \beta$-induced arthritis via reduction in Bax and p53 phosphorylation as well as an increase in $\mathrm{Bcl}-\mathrm{xL}$ expression. Additionally, Ecd inhibited NF- $\kappa \mathrm{B}$ phosphorylation, $\mathrm{I} \kappa \mathrm{B} \alpha$ degradation, and MMP-3, MMP-9, and COX-2 expression in IL- $1 \beta$-induced arthritis. Ecd is a potent herbal medicine, used for its antiapoptotic and anti-inflammatory properties in OA (Table 2) [90]. Thrombin activity was measured by aqueous extracts from Ceiba pentandra (Malvaceae/Bombacoideae) and Quassia africana (Simaroubaceae) (C. pentandra). The results indicated the reduction of thrombin activity and prolonged plasma clotting time through affecting coagulation of intrinsic pathway [91]. Although there is no direct evidence that herbal medicines target thrombin in degenerative arthritis, the above results indicate both reduction of thrombin activity and extension of plasma clotting time, thus affecting the intrinsic coagulation pathway. Therefore, herbal medicines may have an adjunct nutraceutical chondroprotective application in treatment of $\mathrm{OA}$ and related osteoarticular disorders.

\section{Conclusion}

To conclude, we summarize that thrombin is a key factor in stimulation of fibrin deposition and proinflammatory processes in OA. Therefore, study of the thrombin signaling pathway helps us elucidate the mechanism underlying OA pathogenesis and can lead to novel therapeutic strategies in OA. Treatments that can inhibit thrombin expression and related PARs are the focus of clinical and preclinical 
studies that are currently underway. To date, thrombintargeted treatment for OA has not yet reached the clinicaltrial stage despite the unmet need for effective adjuvant treatments. On the other hand, a recent study showed that angiogenesis could contribute to structural damage and may act as a potential target [92]. Additionally, thrombin was shown to have increased vascular permeability, leading to tissue damage [93]. Thus, development of a specific drug targeting thrombin could inhibit angiogenesis mediators and macrophage-activated inflammatory cytokines could be explored as a therapeutic strategy for OA treatment.

\section{Conflict of Interests}

All authors have no financial or personal relationships with other people or organizations that could inappropriately influence our work.

\section{Acknowledgment}

This work was supported by Grants from Ministry of Science and Technology (MOST 103-2314-B-039-019; 103-2628-B039-002-MY3), China Medical University (CMU102-BC-4). The study sponsors were not involved in the study design, data collection or analysis, or in the writing of the paper.

\section{References}

[1] K. D. Brandt, P. Dieppe, and E. Radin, "Etiopathogenesis of osteoarthritis," Medical Clinics of North America, vol. 93, no. 1, pp. 1-24, 2009.

[2] D. T. Felson, "Epidemiology of hip and knee osteoarthritis," Epidemiologic Reviews, vol. 10, pp. 1-28, 1988.

[3] R. Altman, G. Alarcón, D. Appelrouth et al., "The American college of rheumatology criteria for the classification and reporting of osteoarthritis of the hip," Arthritis and Rheumatism, vol. 34, no. 5, pp. 505-514, 1991.

[4] S. P. Oakley, M. N. Lassere, I. Portek et al., "Biomechanical, histologic and macroscopic assessment of articular cartilage in a sheep model of osteoarthritis," Osteoarthritis and Cartilage, vol. 12, no. 8, pp. 667-679, 2004.

[5] L. Sharma, C. Lou, S. Cahue, and D. D. Dunlop, "The mechanism of the effect of obesity in knee osteoarthritis: the mediating role of malalignment," Arthritis \& Rheumatology, vol. 43, no. 3, pp. 568-575, 2000.

[6] F. W. Bora Jr. and G. Miller, "Joint physiology, cartilage, metabolism, and the etiology of osteoarthritis," Hand Clinics, vol. 3, no. 3, pp. 325-336, 1987.

[7] A. Lahm, E. Mrosek, H. Spank et al., "Changes in content and synthesis of collagen types and proteoglycans in osteoarthritis of the knee joint and comparison of quantitative analysis with Photoshop-based image analysis," Archives of Orthopaedic and Trauma Surgery, vol. 130, no. 4, pp. 557-564, 2010.

[8] F. A. Wollheim, "Serum markers of articular cartilage damage and repair," Rheumatic Disease Clinics of North America, vol. 25, no. 2, pp. 417-432, 1999.

[9] M. B. Goldring and K. B. Marcu, "Cartilage homeostasis in health and rheumatic diseases," Arthritis Research and Therapy, vol. 11, no. 3, article 224, 2009.
[10] H. Lorenz and W. Richter, "Osteoarthritis: cellular and molecular changes in degenerating cartilage," Progress in Histochemistry and Cytochemistry, vol. 40, no. 3, pp. 135-163, 2006.

[11] C. Y. Wenham and P. G. Conaghan, "The role of synovitis in osteoarthritis," Therapeutic Advances in Musculoskeletal Disease, vol. 2, no. 6, pp. 349-359, 2010.

[12] A. Thaper, W. Zhang, G. Wright, and M. Doherty, "Relationship between Heberden's nodes and underlying radiographic changes of osteoarthritis," Annals of the Rheumatic Diseases, vol. 64, no. 8, pp. 1214-1216, 2005.

[13] R. F. Loeser Jr., "Aging and the etiopathogenesis and treatment of osteoarthritis," Rheumatic Disease Clinics of North America, vol. 26, no. 3, pp. 547-567, 2000.

[14] J. W. Fenton II, F. A. Ofosu, D. G. Moon, and J. M. Maraganore, "Thrombin structure and function: why thrombin is the primary target for antithrombotics," Blood Coagulation \& Fibrinolysis, vol. 2, no. 1, pp. 69-75, 1991.

[15] J. W. Fenton II, F. A. Ofosu, D. V. Brezniak, and H. Hassouna, "Thrombin and antithrombotics," Seminars in Thrombosis and Hemostasis, vol. 24, no. 2, pp. 87-91, 1998.

[16] S. R. Coughlin, "Thrombin signalling and protease-activated receptors," Nature, vol. 407, no. 6801, pp. 258-264, 2000.

[17] B. Østerud, "Tissue factor expression by monocytes: regulation and pathophysiological roles," Blood Coagulation and Fibrinolysis, vol. 9, no. 1, pp. S9-S14, 1998.

[18] M. I. Bokarewa, J. H. Morrissey, and A. Tarkowski, "Tissue factor as a proinflammatory agent," Arthritis Research, vol. 4, no. 3, pp. 190-195, 2002.

[19] P. F. Neuenschwander, M. M. Fiore, and J. H. Morrissey, "Factor VII autoactivation proceeds via interaction of distinct proteasecofactor and zymogen-cofactor complexes: implications of a two-dimensional enzyme kinetic mechanism," The Journal of Biological Chemistry, vol. 268, no. 29, pp. 21489-21492, 1993.

[20] C. Rubel, S. Gómez, G. C. Fernández, M. A. Isturiz, J. Caamaño, and M. S. Palermo, "Fibrinogen-CD11b/CD18 interaction activates the NF- $\kappa \mathrm{B}$ pathway and delays apoptosis in human neutrophils," European Journal of Immunology, vol. 33, no. 5, pp. 1429-1438, 2003.

[21] D. C. Altieri, F. R. Agbanyo, J. Plescia, M. H. Ginsberg, T. S. Edgington, and E. F. Plow, "A unique recognition site mediates the interaction of fibrinogen with the leukocyte integrin Mac1 (CD11b/CD18)," The Journal of Biological Chemistry, vol. 265, no. 21, pp. 12119-12122, 1990.

[22] O. Sánchez-Pernaute, M. J. Lópezmada, E. Calvo et al., "Fibrin generated in the synovial fluid activates intimal cells from their apical surface: a sequential morphological study in antigeninduced arthritis," Rheumatology, vol. 42, no. 1, pp. 19-25, 2003.

[23] F. M. Szaba and S. T. Smiley, "Roles for thrombin and fibrin(ogen) in cytokine/chemokine production and macrophage adhesion in vivo," Blood, vol. 99, no. 3, pp. 1053-1059, 2002.

[24] M. Nesheim, “Thrombin and fibrinolysis," Chest, vol. 124, no. 3 supplement, pp. 33S-39S, 2003.

[25] C. T. Esmon, “The protein C pathway," Chest, vol. 124, no. 3, pp. 26S-32S, 2003.

[26] H. V. Jakubowski and W. G. Owen, "Macromolecular specificity determinants on thrombin for fibrinogen and thrombomodulin," The Journal of Biological Chemistry, vol. 264, no. 19, pp. 11117-11121, 1989.

[27] L. Bajzar, J. Morser, and M. Nesheim, "TAFI, or plasma procarboxypeptidase $\mathrm{B}$, couples the coagulation and fibrinolytic cascades through the thrombin-thrombomodulin complex," 
The Journal of Biological Chemistry, vol. 271, no. 28, pp. 1660316608, 1996.

[28] S. J. Friezner Degen and E. W. Davie, "Nucleotide sequence of the gene for human prothrombin," Biochemistry, vol. 26, no. 19, pp. 6165-6177, 1987.

[29] M. Andrew, B. Paes, R. Milner et al., "Development of the human coagulation system in the full-term infant," Blood, vol. 70, no. 1, pp. 165-172, 1987.

[30] M. D. Hollenberg and S. J. Compton, "International Union of Pharmacology. XXVIII. Proteinase-activated receptors," Pharmacological Reviews, vol. 54, no. 2, pp. 203-217, 2002.

[31] F. Gieseler, H. Ungefroren, U. Settmacher, M. D. Hollenberg, and R. Kaufmann, "Proteinase-activated receptors (PARs) focus on receptor-receptor- interactions and their physiological and pathophysiological impact," Cell Communication and Signaling, vol. 11, no. 1, article 86, 2013.

[32] R. Ramachandran, F. Noorbakhsh, K. Defea, and M. D. Hollenberg, "Targeting proteinase-activated receptors: therapeutic potential and challenges," Nature Reviews Drug Discovery, vol. 11, no. 1, pp. 69-86, 2012.

[33] M. Steinhoff, J. Buddenkotte, V. Shpacovitch et al., "Proteinaseactivated receptors: transducers of proteinase-mediated signaling in inflammation and immune response," Endocrine Reviews, vol. 26, no. 1, pp. 1-43, 2005.

[34] S. R. Coughlin, "Protease-activated receptors in hemostasis, thrombosis and vascular biology," Journal of Thrombosis and Haemostasis, vol. 3, no. 8, pp. 1800-1814, 2005.

[35] S. F. Traynelis and J. Trejo, "Protease-activated receptor signaling: new roles and regulatory mechanisms," Current Opinion in Hematology, vol. 14, no. 3, pp. 230-235, 2007.

[36] G. R. Sambrano, W. Huang, T. Faruqi, S. Mahrus, C. Craik, and S. R. Coughlin, "Cathepsin G activates protease-activated receptor-4 in human platelets," The Journal of Biological Chemistry, vol. 275, no. 10, pp. 6819-6823, 2000.

[37] T. R. Faruqi, E. J. Weiss, M. J. Shapiro, W. Huang, and S. R. Coughlin, "Structure-function analysis of protease-activated receptor 4 thetered Ligand peptides. Determinants of specificity and utility in assays of receptor function," The Journal of Biological Chemistry, vol. 275, no. 26, pp. 19728-19734, 2000.

[38] K. A. DeFea, J. Zalevsky, M. S. Thoma, O. Dery, R. D. Mullins, and N. W. Bunnett, " $\beta$-Arrestin-dependent endocytosis of proteinase-activated receptor 2 is required for intracellular targeting of activated ERK1/2," Journal of Cell Biology, vol. 148, no. 6, pp. 1267-1281, 2000.

[39] H. Lum and A. B. Malik, "Regulation of vascular endothelial barrier function," American Journal of Physiology, vol. 267, no. 3, part 1, pp. L223-L241, 1994.

[40] G. P. van Nieuw Amerongen, S. V. Delft, M. A. Vermeer, J. G. Collard, and V. W. M. van Hinsbergh, "Activation of RhoA by thrombin in endothelial hyperpermeability: role of rho kinase and protein tyrosine kinases," Circulation Research, vol. 87, no. 4, pp. 335-340, 2000.

[41] L. Zhu, X. Wang, J. Wu et al., "Cooperation of protease-activated receptor 1 and integrin $\alpha \mathrm{v} \beta 5$ in thrombin-mediated lung cancer cell invasion," Oncology Reports, vol. 28, no. 2, pp. 553-560, 2012.

[42] D. Fujimoto, Y. Hirono, T. Goi, K. Katayama, S. Matsukawa, and A. Yamaguchi, "The activation of proteinase-activated receptor1 (PAR1) promotes gastric cancer cell alteration of cellular morphology related to cell motility and invasion," International Journal of Oncology, vol. 42, no. 2, pp. 565-573, 2013.
[43] D. Darmoul, V. Gratio, H. Devaud, T. Lehy, and M. Laburthe, "Aberrant expression and activation of the thrombin receptor protease-activated receptor-1 induces cell proliferation and motility in human colon cancer cells," American Journal of Pathology, vol. 162, no. 5, pp. 1503-1513, 2003.

[44] R. Colognato, J. R. Slupsky, M. Jendrach, L. Burysek, T. Syrovets, and T. Simmet, "Differential expression and regulation of protease-activated receptors in human peripheral monocytes and monocyte-derived antigen-presenting cells," Blood, vol. 102, no. 7, pp. 2645-2652, 2003.

[45] A. Naldini, A. Pucci, D. H. Carney, G. Fanetti, and F. Carraro, "Thrombin enhancement of interleukin-1 expression in mononuclear cells: involvement of proteinase-activated receptor-1," Cytokine, vol. 20, no. 5, pp. 191-199, 2002.

[46] A. Naldini, C. Bernini, A. Pucci, and F. Carraro, "Thrombinmediated IL-10 up-regulation involves protease-activated receptor (PAR)-1 expression in human mononuclear leukocytes," Journal of Leukocyte Biology, vol. 78, no. 3, pp. 736-744, 2005.

[47] A. Stankovic, V. Slavic, B. Stamenkovic, B. Kamenov, M. Bojanovic, and D. R. Mitrovic, "Serum and synovial fluid concentrations of CCL2 (MCP-1) chemokine in patients suffering rheumatoid arthritis and osteoarthritis reflect disease activity," Bratislavské Lekárske Listy, vol. 110, no. 10, pp. 641-646, 2009.

[48] C. Y. Huang, S. Y. Chen, H. C. Tsai, H. C. Hsu, and C. H. Tang, "Thrombin induces epidermal growth factor receptor transactivation and CCL2 expression in human osteoblasts," Arthritis and Rheumatism, vol. 64, no. 10, pp. 3344-3354, 2012.

[49] J. J. Oppenheim, C. O. C. Zachariae, N. Mukaida, and K. Matsushima, "Properties of the novel proinflammatory supergene "intercrine" cytokine family," Annual Review of Immunology, vol. 9, no. 1, pp. 617-648, 1991.

[50] R. E. Miller, P. B. Tran, R. Das et al., "CCR2 chemokine receptor signaling mediates pain in experimental osteoarthritis," Proceedings of the National Academy of Sciences of the United States of America, vol. 109, no. 50, pp. 20602-20607, 2012.

[51] M. Duarte, V. Kolev, R. Soldi et al., “Thrombin induces rapid PAR1-mediated non-classical FGF1 release," Biochemical and Biophysical Research Communications, vol. 350, no. 3, pp. 604609, 2006.

[52] A. Cucina, V. Borrelli, A. Di Carlo et al., "Thrombin induces production of growth factors from aortic smooth muscle cells," Journal of Surgical Research, vol. 82, no. 1, pp. 61-66, 1999.

[53] Y. Xiang, K. Masuko-Hongo, T. Sekine et al., "Expression of proteinase-activated receptors (PAR)-2 in articular chondrocytes is modulated by IL- $1 \beta$, TNF- $\alpha$ and TGF- $\beta$," Osteoarthritis and Cartilage, vol. 14, no. 11, pp. 1163-1173, 2006.

[54] Y. Kirilak, N. J. Pavlos, C. R. Willers et al., "Fibrin sealant promotes migration and proliferation of human articular chondrocytes: possible involvement of thrombin and protease-activated receptors," International Journal of Molecular Medicine, vol. 17, no. 4, pp. 551-558, 2006.

[55] S. J. Song, C. N. Pagel, T. M. Campbell, R. N. Pike, and E. J. Mackie, "The role of protease-activated receptor-1 in bone healing," The American Journal of Pathology, vol. 166, no. 3, pp. 857-868, 2005.

[56] E. Furmaniak-Kazmierczak, T. D. V. Cooke, R. Manuel et al., "Studies of thrombin-induced proteoglycan release in the degradation of human and bovine cartilage," The Journal of Clinical Investigation, vol. 94, no. 2, pp. 472-480, 1994.

[57] Y.-C. Chiu, R.-S. Yang, K.-H. Hsieh et al., "Stromal cellderived factor-1 induces matrix metalloprotease- 13 expression 
in human chondrocytes," Molecular Pharmacology, vol. 72, no. 3, pp. 695-703, 2007.

[58] M. Wang, E. R. Sampson, H. Jin et al., "MMP13 is a critical target gene during the progression of osteoarthritis," Arthritis Research \& Therapy, vol. 15, no. 1, article R5, 2013.

[59] T. Shiomi, V. Lemaître, J. D’Armiento, and Y. Okada, "Matrix metalloproteinases, a disintegrin and metalloproteinases, and a disintegrin and metalloproteinases with thrombospondin motifs in non-neoplastic diseases: review Article," Pathology International, vol. 60, no. 7, pp. 477-496, 2010.

[60] C. Y. Huang, H. J. Lin, H. S. Chen, S. Y. Cheng, H. C. Hsu, and C. H. Tang, "Thrombin promotes matrix metalloproteinase-13 expression through the PKCdelta c-Src/EGFR/PI3K/Akt/AP1 signaling pathway in human chondrocytes," Mediators of Inflammation, vol. 2013, Article ID 326041, 12 pages, 2013.

[61] G. Kikuchi, T. Yoshida, and M. Noguchi, "Heme oxygenase and heme degradation," Biochemical and Biophysical Research Communications, vol. 338, no. 1, pp. 558-567, 2005.

[62] S. W. Ryter and A. M. K. Choi, "Heme oxygenase-1: molecular mechanisms of gene expression in oxygen-related stress," Antioxidants and Redox Signaling, vol. 4, no. 4, pp. 625-632, 2002.

[63] M. J. Alcaraz, P. Fernandez, and M. I. Guillén, "Antiinflammatory actions of the heme oxygenase-1 pathway," Current Pharmaceutical Design, vol. 9, no. 30, pp. 2541-2551, 2003.

[64] I. Devesa, M. L. Ferrándiz, M. C. Terencio, L. A. B. Joosten, W. B. van den Berg, and M. J. Alcaraz, "Influence of heme oxygenase 1 modulation on the progression of murine collagen-induced arthritis," Arthritis and Rheumatism, vol. 52, no. 10, pp. 32303238, 2005.

[65] H. A. Kim, K. B. Lee, and S.-C. Bae, "The mechanism of low-concentration sodium nitroprusside-mediated protection of chondrocyte death," Arthritis research \& therapy., vol. 7, no. 3, pp. R526-R535, 2005.

[66] Y. Henrotin, L. Pesesse, and C. Lambert, "Targeting the synovial angiogenesis as a novel treatment approach to osteoarthritis," Therapeutic Advances in Musculoskeletal Disease, vol. 6, no. 1, pp. 20-34, 2014.

[67] Y. Kitamoto, E. Nakamura, S. Kudo et al., "Thrombin in synovial fluid as a marker of synovial inflammation: a definite measurement by ELISA and correlation with VEGF," Clinica Chimica Acta, vol. 398, no. 1-2, pp. 159-160, 2008.

[68] M. J. Benito, D. J. Veale, O. FitzGerald, W. B. van den Berg, and B. Bresnihan, "Synovial tissue inflammation in early and late osteoarthritis," Annals of the Rheumatic Diseases, vol. 64, no. 9, pp. 1263-1267, 2005.

[69] M. D. Smith, S. Triantafillou, A. Parker, P. P. Youssef, and M. Coleman, "Synovial membrane inflammation and cytokine production in patients with early osteoarthritis," Journal of Rheumatology, vol. 24, no. 2, pp. 365-371, 1997.

[70] H.-L. Hsieh, C.-C. Sun, T.-S. Wang, and C.-M. Yang, "PKC- $\delta / c-$ Src-mediated EGF receptor transactivation regulates thrombininduced COX-2 expression and PGE2 production in rat vascular smooth muscle cells," Biochimica et Biophysica Acta, vol. 1783, no. 9, pp. 1563-1575, 2008.

[71] M. Yeh, N. M. Gharavi, J. Choi et al., "Oxidized phospholipids increase interleukin 8 (IL-8) synthesis by activation of the csrc/signal transducers and activators of transcription (STAT) 3 pathway," The Journal of Biological Chemistry, vol. 279, no. 29, pp. 30175-30181, 2004.

[72] T. Ishii, K. Itoh, E. Ruiz et al., "Role of Nrf2 in the regulation of CD36 and stress protein expression in murine macrophages: activation by oxidatively modified LDL and 4-hydroxynonenal," Circulation Research, vol. 94, no. 5, pp. 609-616, 2004.

[73] A. K. So, P.-A. Varisco, B. Kemkes-Matthes et al., "Arthritis is linked to local and systemic activation of coagulation and fibrinolysis pathways," Journal of Thrombosis and Haemostasis, vol. 1, no. 12, pp. 2510-2515, 2003.

[74] P. A. Varisco, V. Péclat, K. Van Ness, A. Bischof-Delaloye, A. So, and N. Busso, "Effect of thrombin inhibition on synovial inflammation in antigen induced arthritis," Annals of the Rheumatic Diseases, vol. 59, no. 10, pp. 781-787, 2000.

[75] M. di Nisio, S. Middeldorp, and H. R. Büller, "Direct thrombin inhibitors," The New England Journal of Medicine, vol. 353, no. 10, pp. 1028-1040, 2005.

[76] C. J. Lee and J. E. Ansell, "Direct thrombin inhibitors," British Journal of Clinical Pharmacology, vol. 72, no. 4, pp. 581-592, 2011.

[77] H. Nar, "The role of structural information in the discovery of direct thrombin and factor Xa inhibitors," Trends in Pharmacological Sciences, vol. 33, no. 5, pp. 279-288, 2012.

[78] J. I. Weitz and M. Crowther, "Direct thrombin inhibitors," Thrombosis Research, vol. 106, no. 3, pp. V275-V284, 2002.

[79] D. J. Angiolillo, D. Capodanno, and S. Goto, "Platelet thrombin receptor antagonism and atherothrombosis," European Heart Journal, vol. 31, no. 1, pp. 17-28, 2010.

[80] S. Chackalamannil, “Thrombin receptor (protease activated receptor-1) antagonists as potent antithrombotic agents with strong antiplatelet effects," Journal of Medicinal Chemistry, vol. 49, no. 18, pp. 5389-5403, 2006.

[81] D. J. Fintel, "Oral antiplatelet therapy for atherothrombotic disease: overview of current and emerging treatment options," Vascular Health and Risk Management, vol. 8, no. 1, pp. 77-89, 2012.

[82] V. L. Serebruany, M. Kogushi, D. Dastros-Pitei, M. Flather, and D. L. Bhatt, "The in-vitro effects of E5555, a proteaseactivated receptor (PAR)-1 antagonist, on platelet biomarkers in healthy volunteers and patients with coronary artery disease," Thrombosis \& Haemostasis, vol. 102, no. 1, pp. 111-119, 2009.

[83] J. W. Fenton, G. B. Villanueva, F. A. Ofosu, and J. M. Maraganore, "Thrombin inhibition by hirudin: how hirudin inhibits thrombin," Haemostasis, vol. 21, no. 1, pp. 27-31, 1991.

[84] G. Cirino, C. Cicala, M. R. Bucci, L. Sorrentino, J. M. Maraganore, and S. R. Stone, "Thrombin functions as an inflammatory mediator through activation of its receptor," Journal of Experimental Medicine, vol. 183, no. 3, pp. 821-827, 1996.

[85] M. A. Cunningham, E. Rondeau, X. Chen, S. R. Coughlin, S. R. Holdsworth, and P. G. Tipping, "Protease-activated receptor 1 mediates thrombin-dependent, cell-mediated renal inflammation in crescentic glomerulonephritis," Journal of Experimental Medicine, vol. 191, no. 3, pp. 455-461, 2000.

[86] R. J. MacFarlane, S. M. Graham, P. S. E. Davies et al., "Antiinflammatory role and immunomodulation of mesenchymal stem cells in systemic joint diseases: potential for treatment," Expert Opinion on Therapeutic Targets, vol. 17, no. 3, pp. 243254, 2013.

[87] M. Kapoor, J. Martel-Pelletier, D. Lajeunesse, J.-P. Pelletier, and H. Fahmi, "Role of proinflammatory cytokines in the pathophysiology of osteoarthritis," Nature Reviews Rheumatology, vol. 7, no. 1, pp. 33-42, 2011.

[88] M. Shakibaei, G. Schulze-Tanzil, T. John, and A. Mobasheri, "Curcumin protects human chondrocytes from IL- $1 \beta$-induced inhibition of collagen type II and $\beta 1$-integrin expression and activition of caspase-3: an immunomorphological study," Annals of Anatomy, vol. 187, no. 5-6, pp. 487-497, 2005. 
[89] Y. Panahi, A. R. Rahimnia, M. Sharafi, G. Alishiri, A. Saburi, and A. Sahebkar, "Curcuminoid treatment for knee osteoarthritis: a randomized double-Blind Placebo-controlled trial," Phytotherapy Research, 2014.

[90] X. Zhang, X. Xu, T. Xu, and S. Qin, “ $\beta$-ecdysterone suppresses interleukin- $1 \beta$-induced apoptosis and inflammation in rat chondrocytes via inhibition of NF- $\kappa \mathrm{B}$ signaling pathway," Drug Development Research, vol. 75, no. 3, pp. 195-201, 2014.

[91] M. M. Nsimba, J. N. Lami, Y. Hayakawa, C. Yamamoto, and T. Kaji, "Decreased thrombin activity by a Congolese herbal medicine used in sickle cell anemia," Journal of Ethnopharmacology, vol. 148, no. 3, pp. 895-900, 2013.

[92] P. I. Mapp and D. A. Walsh, "Mechanisms and targets of angiogenesis and nerve growth in osteoarthritis," Nature Reviews Rheumatology, vol. 8, no. 7, pp. 390-398, 2012.

[93] J. M. Siller-Matula, M. Schwameis, A. Blann, C. Mannhalter, and B. Jilma, "Thrombin as a multi-functional enzyme: focus on in vitro and in vivo effects," Thrombosis and Haemostasis, vol. 106, no. 6, pp. 1020-1033, 2011. 

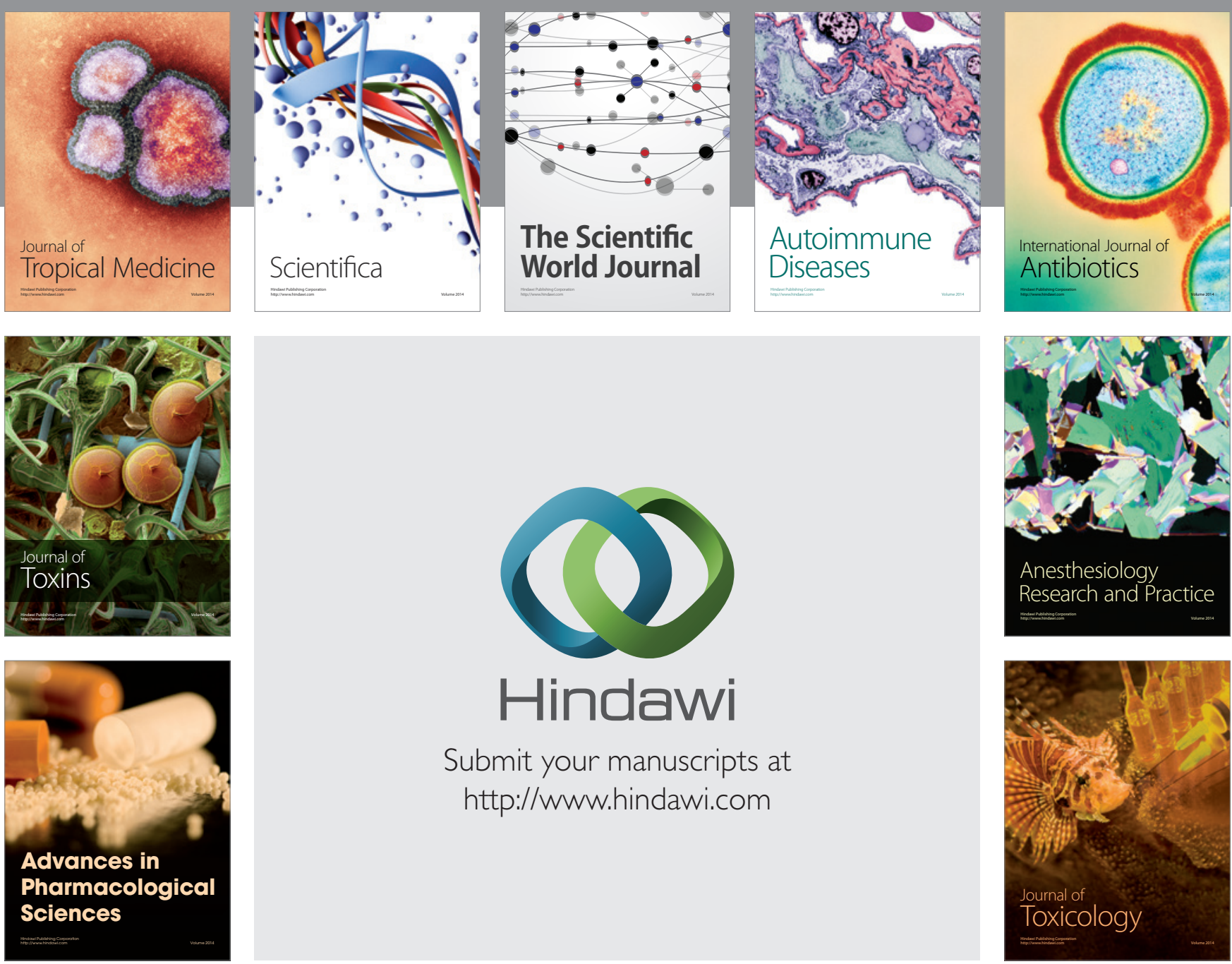

\section{Hindawi}

Submit your manuscripts at

http://www.hindawi.com
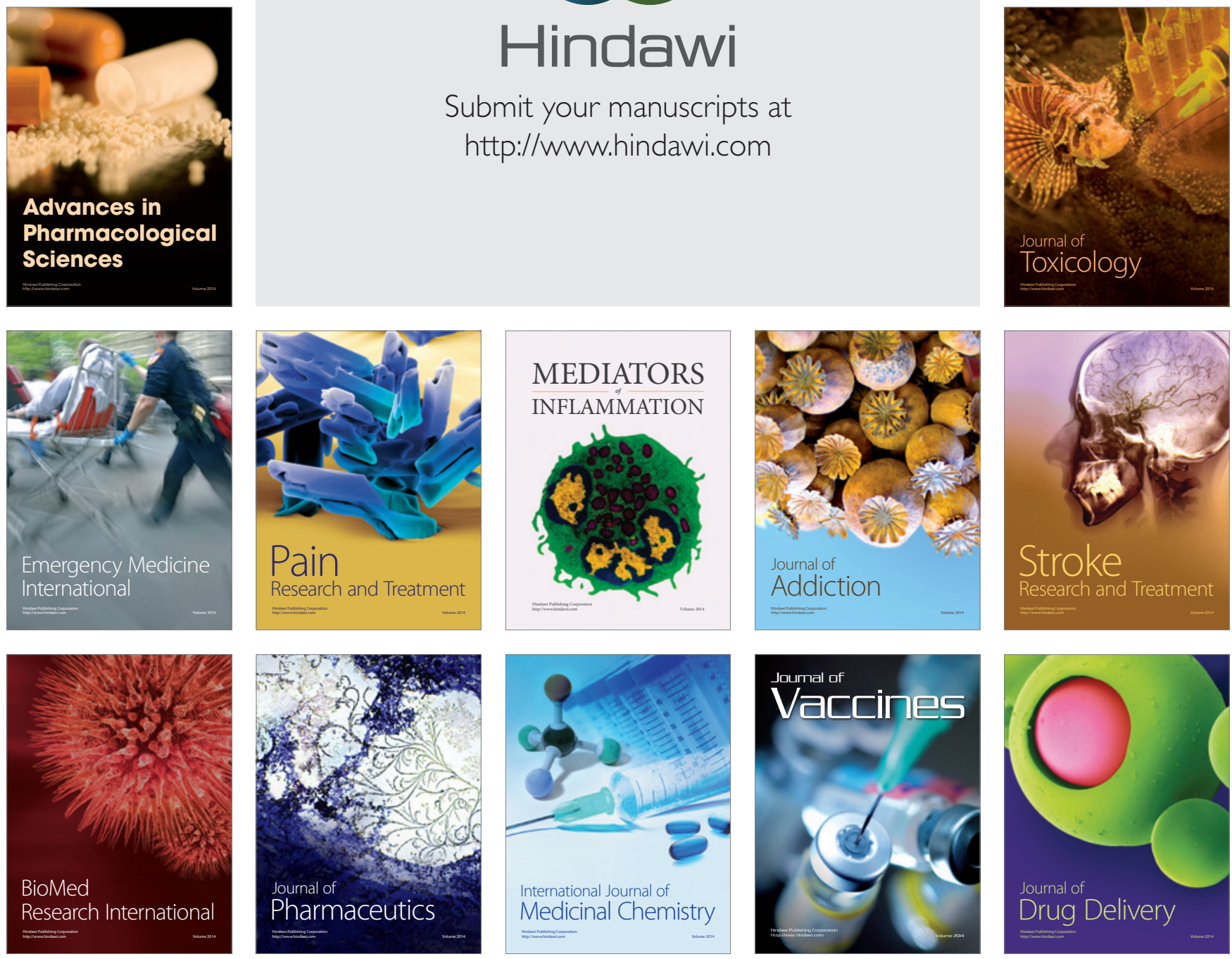